\title{
Zinc Oxide Nanorods Wrapped with Ion-Imprinted Polypyrrole Polymer for Picomolar Selective and Electrochemical Detection of Mercury II Ions ${ }^{+}$
}

\author{
Zouhair Ait-Touchente 1,*, Houssem Eddine El Yamine Sakhraoui ${ }^{2}$, Najla Fourati ${ }^{3}$, \\ Chouki Zerrouki ${ }^{3}$, Naima Maouche ${ }^{2}$, Rachid Touzani ${ }^{1}$, Nourdin Yaakoubi ${ }^{4}$ and \\ Mohamed M. Chehimi ${ }^{5}$ \\ 1 LCAE-FSO, Université Mohammed Premier, Oujda 60000, Morocco; touzanir@yahoo.fr \\ 2 Laboratoire d'Electrochimie et Matériaux, Université Ferhat Abbas, Sétif-1 19000, Algeria; \\ h_sakhraoui@hotmail.com (H.E.E.Y.S.) ;-nmaouche@univ-setif.dz (N.M.) \\ 3 SATIE, UMR CNRS 8029, Cnam, 75003 Paris, France; fourati@cnam.fr (N.F.) ; zerrouki@cnam.fr (C.Z.) \\ 4 LAUM, UMR CNRS 6613, Le Mans Univrsité, 72085 Le Mans, France; nourdin.yaakoubi@univ-lemans.fr \\ 5 ICMPE, UMR CNRS 7182, CNRS, 94320 Thiais, France; mmchehimi@yahoo.fr \\ * Correspondence: zouhair.aittouchente@gmail.com; Tel.: +33-1-58-80-87-03 \\ † Presented at the Eurosensors 2018 Conference, Graz, Austria, 9-12 September 2018. \\ Published: 5 December 2018
}

\begin{abstract}
This study concerns the design of an ion-imprinted polymer (IIP) for the selective detection of mercury II ions. Compared to other electrochemical studies, the originality of this work lies to the fact that the IIP was electropolymerized on $\mathrm{ZnO}$ nanorods, which were themselves grown on gold/diazonium modified substrates. This strategy of diazonium salt and $\mathrm{ZnO}$ nanorods permits to increase considerably the specific surface and thus to improve the sensor's performances. The limit of detection (LOD) of the designed sensor was of order of $1 \mathrm{pM}$, the lowest value ever reported in literature.
\end{abstract}

Keywords: ionic imprinted polymer; electrochemical detection; mercury; zinc oxide nanorods

\section{Introduction}

As other heavy metal ions, mercury (II) presents health and ecological concerns [1]. Its presence in the ecosystem is related to its wide use in cement kilns, gold mining and trash incinerators [2]. Exposure to mercury ions, even at low doses, can lead to severe central nervous system problems and vital human organs damage [3,4]. The liberation of $\mathrm{Hg}$ (II) in the environment is forbidden by the European Union and World Health Organization (WHO) [5]. Its maximum permissible limit in drinking water is equal to $2 \mu \mathrm{g} / \mathrm{L}$, according to the WHO. Developing reliable, sensitive and selective methods for the determination of low concentrations of mercury in food and drinking water is thus of significance.

Due to their high selectivity, sensitivity and low cost, electrochemical transducers functionalized with ion imprinted polymers (IIP) are increasingly used for heavy ions detection in both simple and complex media. In most cases, IIPs are deposited directly on the surface of a bare electrode [6]. Here, we have chosen to electropolymerize the IIP on $\mathrm{ZnO}$ nanorods, which are themselves grown on a gold electrode. The ultimate goal was an increase in the sensor's sensitivity and a diminution of the limit of detection (LOD). 


\section{Materials and Methods}

\subsection{Reagents}

4-Aminobenzoic acid (99\%), methanol (MeOH), $\mathrm{H}_{2} \mathrm{SO}_{4}(95 \%) \mathrm{H}_{2} \mathrm{O}_{2}(30 \%)$, tetrabutylammonium tetrafluoroborate $(99 \%)$, zinc acetate dihydrate $(99.99 \%)$, sodium hydroxide $(97 \%)$, hexamethylenetetramine $(99 \%)$, acetonitrile $(99.8 \%)$, potassium hexacyanoferrate(III) $(99 \%)$, potassium hexacyanoferrate(II) trihydrate $(98.5 \%)$, potassium chloride, pyrrole (Py) $(98 \%)$, lead nitrate $\mathrm{Pb}\left(\mathrm{NO}_{3}\right)_{2}$, copper(II) nitrate hemi(pentahydrate) $(98 \%)$ and mercury (II) chloride $(98 \%)$ were purchased from Sigma-Aldrich. Pyrrole was purified through alumina basic column and stored in dark at $4^{\circ} \mathrm{C}$. Cadmium sulfate $8 / 3$-hydrate $(99 \%)$ was supplied by VWR Prolabo. Zinc nitrate hexahydrate (99\%) was obtained from Merck, isopentyl nitrite (97\%) was supplied by Alfa Aesar. All solvents were of analytical grade and all aqueous solutions were prepared using ultrapure milli-Q water.

\subsection{Instruments and Characterization}

Electrochemical measurements were carried out with a PG581 Bio-Logic portable potentiostat. Conventional three-electrodes cell was used, with $\mathrm{Ag} / \mathrm{AgCl}$, a platinum grid, and a gold substrate as the reference, counter and working electrodes, respectively.

\subsection{Surface Functionalization by Diazonium Salt}

The surface modification of bare gold electrodes was carried out with 4carboxybenzenediazonium tetrafluoroborate, which was in-situ generated using 4-aminobenzoicacid [7]. Electroreduction of diazonium salt on gold electrodes was performed by cyclic voltammetry at a scanning rate of $100 \mathrm{mV} / \mathrm{s}$ between -1 and $0.0 \mathrm{~V}$ versus $\mathrm{Ag} / \mathrm{AgCl}$ in a solution of acetonitrile containing $1 \mathrm{mM}$ diazonium salt and $0.1 \mathrm{M}$ tetrabutylammonium tetrafluoroborate. The modified electrodes were then rinsed with ultrapure water, ethanol and dried. Results (not shown here) indicate that 20, is the optimum number of cycles.

\subsection{Synthesis, Deposition and $\mathrm{ZnO}$ Growth}

A two-step process has been followed to grow $\mathrm{ZnO}$ nanorods from $\mathrm{ZnO}$ seed particles at low temperature [8].

Step 1: $0.1 \mathrm{M}$ of zinc acetate and $0.03 \mathrm{M}$ of $\mathrm{NaOH}$ were mixed, in methanol, with a constant stirring at $60^{\circ} \mathrm{C}$ for one hour. The synthesized nanoparticles were then deposited by spin coating on the gold electrodes modified by the diazonium salt. The seeded substrates were after that heated for $1 \mathrm{~h}$ at $125^{\circ} \mathrm{C}$, to insure a good adhesion of the seed particles to the substrate.

Step 2: The gold electrodes with $\mathrm{ZnO}$ nanoparticles were then placed upside down inside a beaker, containing aqueous solutions of $30 \mathrm{mM}$ zinc nitrate hexahydrate and $30 \mathrm{mM}$ hexamethylenetetramine (HMT), and kept at $95^{\circ} \mathrm{C}$ for $5 \mathrm{~h}$ with constant stirring. The gold modified electrodes were finally rinsed with ultra-pure water and dried in an oven preset at $125^{\circ} \mathrm{C}$ for $1 \mathrm{~h}$.

\subsection{IIP and NIP Realization Steps}

A thin polypyrrole barrier layer was first electropolymerized on $\mathrm{Au} / \mathrm{ZnO}$ electrodes to prevent eventual detection of $\mathrm{Hg}^{2+}$ ions by "bare" substrates. IIPs electropolymerization was carried out by chronoamperometry (CA) in a $\mathrm{KCl}$ solution containing $10^{-2} \mathrm{M}$ of purified pyrrole (functional monomer), $10^{-4} \mathrm{M}$ of L-cysteine (cross-linker) and $10^{-2} \mathrm{M}$ of $\mathrm{Hg}^{2+}$ (template). The non-imprinted polymer (NIP) was prepared under the same conditions in the absence of the template.

$\mathrm{Hg}^{2+}$ extraction from the vicinity of the polymeric matrix was done by immersing the electrodes in $0.1 \mathrm{M}$ EDTA solution for $20 \mathrm{~min}$, and then in ultra-pure water for $10 \mathrm{~min}$. 


\section{Results and Discussion}

\subsection{Surface Functionalization via Quasi-Vertical Growth of $\mathrm{ZnO}$}

The electroreduction of the diazonium salt carried out by cyclic voltammetry in 20 cycles gave a virtually zero reduction current (Figure 1A) resulting from a passivation of the electrode following the grafting of aryl groups. The number of cycles was controlled by the ferrocyanide test (Figure 1B) to keep a good surface conductivity. The diazonium salt makes it possible to have an almost homogeneous distribution of quasi vertical growth of $\mathrm{ZnO}$ nanorods.
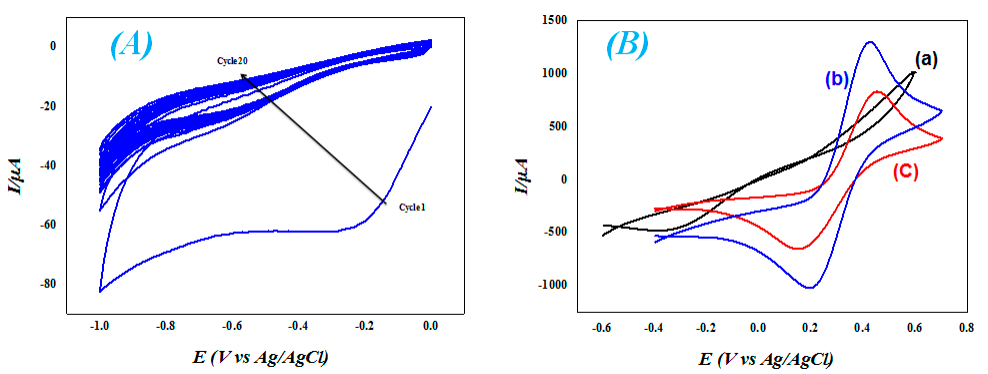

Figure 1. Electro-grafting of 4-carboxybenzenediazonium tetrafluoroborate. (A) Electroreduction, (B) Passivation test: (a): uncleaned gold electrode; (b): cleaned electrode and (c): after electro-grafting of the diazonium salt.

\subsection{Preparation of IIP \& NIP-Based Electrodes}

Both $\mathrm{ZnO}-\mathrm{IIP}$ and the barrier layer were prepared by chronoamperometry (Figure 2A). ZnO-IIP and ZnO-NIP were compared using Square Wave Voltammetry (Figure 2B). Results indicate an obvious difference between the two films, as the characteristic peak current of $\mathrm{Hg}$ (II) is absent in the NIP's response. The extraction of $\mathrm{Hg}^{2+}$ ions from $\mathrm{ZnO}-\mathrm{IIP}$ by EDTA leads to voltammograms close to that of ZnO-NIP (Figure 2B), indicating that all targets were released from the vicinity of the IIP matrix, and that the extracted IIP can be reused for the recapture of the target ions.
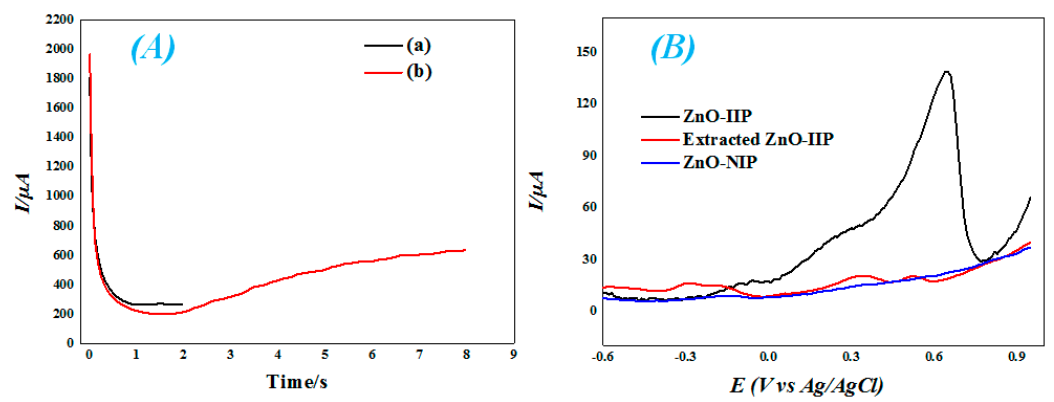

Figure 2. (A): Electropolymerization of pyrrole by chronoamperometry. (a) Barrier layer, (b) Polymerization of pyrrole in the presence of L-cysteine and $\mathrm{Hg}^{2+}$. (B): SWV of IIP and NIP.

\subsection{Electrochemical Sensing of Mercury}

Square wave voltammetry technique was used to investigate the electrochemical response of the $\mathrm{ZnO} / \mathrm{Hg}$ (II)-IIP sensor towards mercury in solution at different concentrations. Sensors response has been recorded, via the characteristic peak current, for concentrations between $10^{-12}$ and $10^{-3} \mathrm{M}$ (Figure $3 \mathrm{~A})$. Up to $10^{-6} \mathrm{M}$, the response exhibits a logarithmic behavior, while a power law is observed for higher concentrations. The sensors limit of detection (LOD) was estimated from the lower accessible concentration, instead of the usual signal to noise ratio. The obtained LOD of $1 \mathrm{pM}$ is largely lower than the WHO maximum permissible limit in drinking water). The sensor's sensitivity, estimated from the slope at the origin of current/concentration curves (Figure 3A), was of order of $7.17 \pm 0.15$ $(\mu \mathrm{A} / \mathrm{M})$. In addition to these interesting metrological features, we also tested the selectivity of the 
realized sensor. This has been done with lead, cadmium and copper ions. Results presented in Figure $3 \mathrm{~B}$ indicates that the developed sensor is selective to $\mathrm{Hg}$ ions, as the mercury peak current (around the characteristic potential) is largely superior to those corresponding to the other ions.
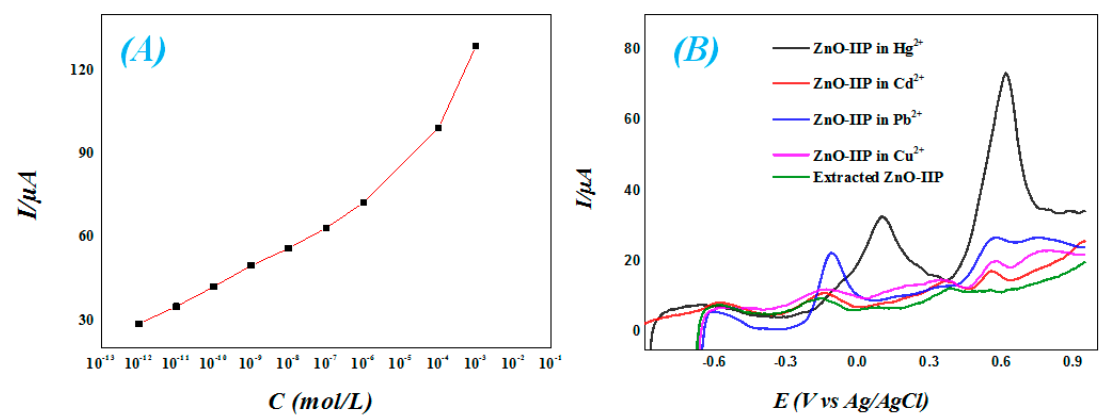

Figure 3. (A): Follow up of pic currents variations (determined from SWV measurements) versus $\mathrm{Hg}^{2+}$ concentration; (B): Selectivity tests: SWV curves of $\mathrm{ZnO} / \mathrm{Hg}$ (II)-IIP electrodes incubated in $10^{-4} \mathrm{M}$ solutions of either mercury, cadmium, lead or copper ions for $20 \mathrm{~min}$.

\section{References}

1. Pacyna, E.G.; Pacyna, J.M. Global emission of mercury from anthropogenic sources in 1995. Water Air Soil Pollut. 2002, 137, 149-165.

2. Hande, P.E.; Samui, A.B.; Kulkarni, P.S. Selective nanomolar detection of mercury using coumarin based fluorescent $\mathrm{Hg}(\mathrm{II})-$ Ion imprinted polymer. Sens. Actuators B Chem. 2017, 246, 597-605.

3. D'Ltri, P.; D'Ltri, F. Mercury contamination: A human tragedy. Environ. Manag. 1978, 2, 3-16.

4. Tchounwou, P.B.; Ayensu, W.K.; Ninashvili, N.; Sutton, D. Review: Environmental exposure to mercury and its toxicopathologic implications for public health. Environ. Toxicol. 2003, 18, 149-175.

5. Chen, G.; Guo, Z.; Zeng, G.; Tang, L. Fluorescent and colorimetric sensors for environmental mercury detection. Analyst 2015, 140, 5400-5443.

6. Martín-Yerga, D.; González-García, M.B.; Costa-García, A. Electrochemical determination of mercury: A review. Talanta 2013, 116, 1091-1104.

7. Li, Z.-C.; Fan, H.-T.; Zhang, Y.; Chen, M.-X.; Yu, Z.-Y.; Cao, X.-Q.; Sun, T. Cd(II)-imprinted polymer sorbents prepared by combination of surface imprinting technique with hydrothermal assisted sol-gel process for selective removal of cadmium(II) from aqueous solution. Chem. Eng. J. 2011, 171, 703-710.

8. Mekki, A.; Ait-Touchente, Z.; Samanta, S.; Singh, A.; Mahmoud, R.; Chehimi, M.M.; Aswal, D.K. PolyanilineWrapped ZnO Nanorod Composite Films on Diazonium-Modified Flexible Plastic Substrates. Macromol. Chem. Phys. 2016, 217, 1136-1148.

(C) 2018 by the authors. Licensee MDPI, Basel, Switzerland. This article is an open access article distributed under the terms and conditions of the Creative Commons Attribution (CC BY) license (http://creativecommons.org/licenses/by/4.0/). 